\title{
Factores asociados a dehiscencia en cáncer de colon
}

\author{
Risk factors of anastomotic leakage in colon cancer
}

Francisco M. González-Valverde ${ }^{1 *}$, María Vicente-Ruiz ${ }^{2}$ y María J. Gómez-Ramos ${ }^{3}$

${ }^{1}$ Departamento de cirugía, Universidad de Murcia. Servicio de Cirugía General y Aparato Digestivo, Hospital General Universitario Reina Sofía; ${ }^{2}$ Servicio de Cirugía General y Aparato Digestivo, Hospital Rafael Méndez, Lorca; ${ }^{3}$ Servicio de Medicina Intensiva, Hospital General Universitario Reina Sofía. Murcia, España

\section{Resumen}

Objetivo: Analizar los posibles factores de riesgo de dehiscencia de anastomosis tras cirugía de cáncer de colon en nuestro entorno y elaborar una ecuación predictiva del riesgo. Método: Estudio de casos y controles sobre una cohorte de 576 pacientes intervenidos de cáncer de colon. Se realizó análisis descriptivo, análisis univariante y regresión logística multivariante para la predicción del riesgo de dehiscencia de anastomosis mediante una ecuación predictiva asociada a curva ROC. Resultados: Existe mayor riesgo de presentar dehiscencia de anastomosis cuando el tiempo quirúrgico supera los 180 minutos. La transfusión preoperatoria, la patología previa, el estado nutricional, la vía de abordaje, la técnica quirúrgica y la edad no influyen en el desarrollo de esta complicación. Se ha determinado el punto de corte óptimo para la predicción aplicando la ecuación, que presenta una sensibilidad del 64.1\% y una especificidad del 67.5\%. Conclusión: El tiempo quirúrgico prolongado es el principal factor de riesgo de fuga tras la cirugía. Nuestra ecuación difícilmente puede predecir dicho riesgo. Tras su validación, nuestros resultados pueden ayudar al cirujano a tomar una decisión individualizada y segura sobre realizar una anastomosis primaria o dejar un estoma.

PALABRAS CLAVE: Cáncer de colon. Dehiscencia de anastomosis. Ecuación predictiva. Factores de riesgo.

\begin{abstract}
Objective: To analyze the risk factors for anastomosis leak in colon cancer surgery (CCS) in our environment, and developed a predictive equation for that risk. Method: We performed a case-control study nested in a cohort of 576 consecutive patients undergoing colon cancer surgery with primary anastomosis, univariate statistical tests and univariate logistic regression for statistical analysis of associated factors with anastomosis leak in colon cancer surgery, and multivariate logistic regression for predicting that risk using a predictive equation associated with a ROC curve. Results: We obtained a higher risk of anastomosis leak in patients whose operative time was longer than 180 minutes. The variables: preoperative transfusion, previous pathologies, nutritional status, approach, surgical technique or age do not influence the development of this complication. The equation found has a sensitivity of $64.1 \%$ and a specificity of $67.5 \%$. Conclusion: Operation time longer than 180 minutes was the main risk factor for anastomosis leak. Our equation can hardly predict this risk. After further validation, our results may help the surgeon make a more individualized, safer decision regarding whether to perform an anastomosis or make a stoma.
\end{abstract}

KEY WORDS: Anastomotic leakage. Colon cancer. Predictive equation. Risk factors.

\author{
Correspondencia: \\ ${ }^{*}$ Francisco M. González-Valverde \\ Victorio, $3,2^{\circ} \mathrm{C}$ \\ C.P. 30003 , Murcia, España \\ E-mail: migova67@gmail.com
}

Fecha de recepción: 22-07-2018

Fecha de aceptación: 10-11-2018

DOI: 10.24875/CIRU.18000616
Cir Cir. 2019;87:347-352

Contents available at PubMed www.cirugiaycirujanos.com 


\section{Introducción}

La dehiscencia de anastomosis tras una resección de colon es una de las complicaciones más importantes y temidas en la cirugía colorrectal. Su incidencia varía del 2 al 4\% en las anastomosis de colon proximal, y del 6 al $12 \%$ en las de tipo distal y extraperitoneal ${ }^{1}$. En 2005, Alves, et al. ${ }^{2}$ demostraron que había una relación negativa en la supervivencia específica del cáncer de colon asociada a esta complicación, y más recientemente Telem, et al. ${ }^{3}$ han publicado cifras de mortalidad cercanas al 10\%. La determinación de los factores de riesgo para dehiscencia de anastomosis es un tema controvertido, como demuestra la existencia de numerosos estudios no concluyentes y con resultados contradictorios. Algunos de los factores estudiados han sido el sexo masculino, el consumo de tabaco, el riesgo en el sistema ASA (American Society of Anesthesiologists), la situación nutricional, el tiempo operatorio prolongado, la pérdida de sangre intraoperatoria y la experiencia del cirujano $0^{1,4}$. No hay un consenso claro del perfil de paciente con riesgo aumentado de sufrir una dehiscencia anastomótica, pero esta complicación sigue ocurriendo con demasiada frecuencia, y aun así es difícil de predecir.

El propósito de este estudio fue analizar los factores de riesgo que podrían influir en el desarrollo de dehiscencia de sutura tras la cirugía de cáncer de colon en nuestro medio, e intentar elaborar un modelo estadístico aplicable que permita predecir esta complicación.

\section{Método}

Se realizó un estudio retrospectivo de casos y controles anidado en una cohorte de base hospitalaria, constituida por todos los pacientes intervenidos de cáncer de colon, para estimar los factores asociados al riesgo de desarrollar dehiscencia de sutura.

La población a estudio estaba formada por todos los pacientes intervenidos quirúrgicamente de cáncer de colon a los que se realizó una resección de colon, abierta o laparoscópica, entre el 1 de enero de 2007 y el 31 de mayo de 2014. En total formaron la muestra 576 pacientes.

La evolución y el seguimiento de estos pacientes se obtuvieron de la historia clínica. Esta selección se realizó a partir de la base de datos del Servicio de Documentación del Hospital General Universitario Reina Sofía, de Murcia (España), tomando los pacientes que recibían el código de cáncer de colon (C18-19) de acuerdo con la Clasificación Internacional de Enfermedades 10. ${ }^{a}$ Revisión. Por cada caso se incorporó un control elegido por muestreo aleatorio simple, quedando un total de 80 pacientes analizados. La definición de caso fue todo paciente intervenido de cáncer de colon en las fechas descritas que había sufrido una dehiscencia de sutura en el posoperatorio. La definición de control fue todo paciente con las mismas características, pero que no hubiera sufrido dehiscencia de sutura. Se excluyeron los pacientes intervenidos de manera urgente y con cáncer de localización rectal. La dehiscencia o disrupción de la anastomosis se definió como la separación de los bordes de sutura que se evidenció por extravasación de contraste radiológico en una prueba de imagen o como una fuga confirmada durante la reintervención quirúrgica'. El protocolo, basado en los principios de la Declaración de Helsinki, fue aprobado por el Comité Ético de Investigación Clínica del Hospital.

\section{Análisis estadístico}

Para determinar las características de la muestra se realizó un análisis descriptivo mediante los estadísticos media, frecuencia y desviación estándar de la muestra. Se analizaron 39 variables dividas en tres grupos: factores preoperatorios o dependientes del paciente, factores de la intervención y factores anatomopatológicos (Tabla 1).

En primer lugar se realizó un análisis utilizando las pruebas t de Student y ji al cuadrado. Se consideró estadísticamente significativo un valor de $p<0.05$. Se realizó un análisis univariante y, a través de un modelo de regresión logística multivariante, usando el procedimiento de pasos hacia atrás para la selección de variables, se estudió la asociación entre la dehiscencia de anastomosis y las variables comentadas. Además, se creó una ecuación predictiva del riesgo de desarrollo de dehiscencia asociado a una curva ROC (Receiver Operating Characteristic) para determinar el punto de corte óptimo y mejorar así la eficacia predictiva del modelo.

Como en todos los estudios retrospectivos, tuvimos la limitación de que aquellas variables no reflejadas en la historia del paciente no siempre pudieron obtenerse en el momento de la recogida de datos. El diseño de casos y controles estuvo sujeto a posibles sesgos de selección y de información que podrían invalidar las conclusiones, por lo que se tomaron medidas para evitar o reducir al mínimo tres tipos de sesgos: la selección sesgada de los pacientes incluidos en el estudio, la obtención de información 
Tabla 1. Variables del estudio

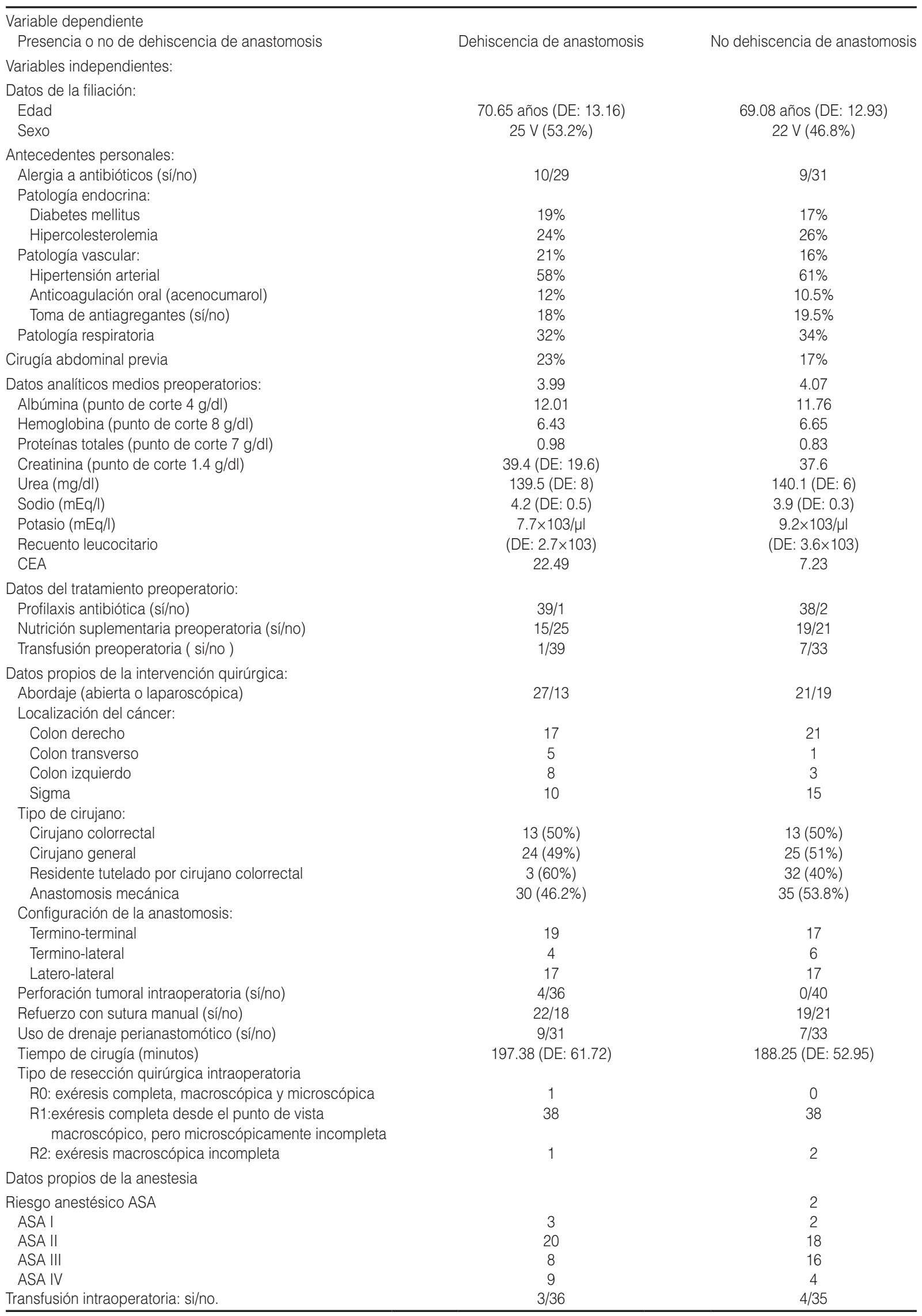

ASA: American Society of Anesthesiologists; CEA: antígeno carcinoembrionario; DE: desviación estándar. 
Tabla 2. Análisis de regresión logística multivariante de factores asociados con la dehiscencia de la anastomosis en cirugía por cáncer de colon

\begin{tabular}{|c|c|c|c|c|c|c|c|c|c|}
\hline & \multirow{2}{*}{$\begin{array}{c}\text { Valores } \\
\text { de las } \\
\text { variables }\end{array}$} & \multirow[t]{2}{*}{ B } & \multirow[t]{2}{*}{ E.T. } & \multirow[t]{2}{*}{ Wald } & \multirow[t]{2}{*}{ gl } & \multirow[t]{2}{*}{ Sig. } & \multirow[t]{2}{*}{ OR } & \multicolumn{2}{|c|}{ IC $95 \%$} \\
\hline & & & & & & & & $\begin{array}{l}\text { Límite } \\
\text { inferior }\end{array}$ & $\begin{array}{l}\text { Límite } \\
\text { superior }\end{array}$ \\
\hline \multirow{2}{*}{$\begin{array}{l}\text { Duración de } \\
\text { la cirugía }\end{array}$} & $<3 h$ & & & & & & 1 & (ref.) & (ref.) \\
\hline & $>3 \mathrm{~h}$ & 1.019 & 0.495 & 4.24 & 1 & 0.039 & 2.77 & 1.05 & 7.307 \\
\hline Creatinina & $<1.4 \mathrm{mg} / \mathrm{dl}$ & & & & & & 1 & (ref.) & (ref.) \\
\hline sérica & $>1.4 \mathrm{mg} / \mathrm{dl}$ & 1.178 & 0.781 & 2.275 & 1 & 0.131 & 3.249 & 0.703 & 15.024 \\
\hline \multirow{2}{*}{$\begin{array}{l}\text { Transfusión } \\
\text { preoperatoria }\end{array}$} & No & & & & & & 1 & (ref.) & (ref.) \\
\hline & Sí & -2.19 & 1.129 & 3.796 & 1 & 0.051 & 0.111 & 0.012 & 1.013 \\
\hline Constante & -1.37 & 0.772 & 3.152 & 1 & 0.076 & 0.254 & & & -1.37 \\
\hline
\end{tabular}

$\mathrm{N}=80 ; \chi^{2}$ del modelo=12.603; $\mathrm{p}=0.006 ;$ Hosmer-Lemeshow $=7.766 ; \mathrm{p}=0.457 ; \mathrm{R}^{2}$ de Nagelkerke $=0.197$.

Variables no incorporadas a la ecuación ( $p$ de incorporación $<0.05$; $p$ de exclusión $>0.010$ ): proteínas, cirugía previa, abordaje, localización, configuración, ASA: American Society of Anesthesiologists.

sesgada y la presencia de factores que pudieran confundir los resultados obtenidos.

\section{Resultados}

Se incluyeron 40 casos y 40 controles, de los cuales $47(58.8 \%)$ eran hombres y $33(41.3 \%)$ mujeres. La edad media de la muestra fue de $69.8 \pm 12.9$ años. Un total de $32(40 \%)$ pacientes fueron intervenidos por laparoscopia, mientras que $45(56.3 \%)$ lo fueron por abordaje abierto. Fue necesario convertir de laparoscopia a laparotomía en $3(3.8 \%)$ casos. La mortalidad fue del $10 \%$ (8 pacientes). Ambos grupos de estudio resultaron homogéneos para sus características basales. En el análisis univariante, dos variables aparecieron asociadas con el desarrollo de dehiscencia de anastomosis: el tiempo operatorio superior a 180 minutos $(p<0.44)$ y la transfusión preoperatoria $(p<0.028)$. El análisis de regresión multivariante (Tabla 2) mostró que la de mayor importancia era el tiempo operatorio superior a 180 minutos (odds ratio [OR]: 2.77), seguida de la transfusión preoperatoria (OR: 0.111). Estas variables, junto a siete más que se consideraron de relevancia clínica en función de la revisión bibliográfica (creatinina sérica $>1.4 \mathrm{mg} / \mathrm{dl}$, proteínas totales preoperatorias, cirugía abdominal previa, vía de abordaje, localización del tumor, configuración de la anastomosis y grado ASA), se utilizaron para crear la ecuación predictiva. De ellas, seis fueron excluidas de la ecuación durante el proceso de selección automática por pasos hacia atrás. Finalmente se llegó a la siguiente ecuación predictiva:

Predicción de dehiscencia de anastomosis (Sí) =Logit $(p)=-1,370+1,019 \times$ (tiempo de duración de la cirugía $>3$ h) $+1.178 \times$ (creatinina sérica $>1.4 \mathrm{mg} / \mathrm{dl})$ $+-2.190 \times$ (transfusión preoperatorio $=$ Sí).

Se obtuvo un punto de corte de 0.5 con una sensibilidad del $64.1 \%$ y una especificidad del $65 \%$. Para mejorar la eficacia del modelo y maximizar la tasa de aciertos en ambos grupos se utilizó el método de curvas ROC, y se obtuvo un punto de corte óptimo de 0.59 con una sensibilidad del $64.1 \%$ y una especificidad aumentada del $67.5 \%$. El valor obtenido para la R cuadrado de Nagelkerke es de 0.197 , por lo que el modelo de regresión logística explica el comportamiento de la variable dependiente dehiscencia de la anastomosis al $19.7 \%$.

\section{Discusión}

El único factor que se ha demostrado estadísticamente significativo para el desarrollo de dehiscencia de anastomosis en el análisis multivariante de este estudio ha sido el tiempo operatorio superior a 180 minutos. Entre los factores anatomopatológicos no se ha encontrado ninguno asociado con un aumento en el riesgo de esta complicación. Sin embargo, la transfusión preoperatoria ha demostrado ser un factor débilmente protector frente a la aparición de dehiscencia de sutura, si bien el resultado se encontraba en el límite de la significación y el hallazgo tiene difícil explicación clínica en la literatura reciente.

Como hemos visto, entre los factores dependientes del paciente, el resultado del análisis multivariante en nuestra serie ha determinado que la creatinina preoperatoria $>1.4 \mathrm{~g} / \mathrm{dl}$ podría ser un factor de riesgo para la aparición de dehiscencia de anastomosis. Sin embargo, a pesar de que la OR es alta, su valor no es 
significativo ( $p=0.131$ ); tiene un amplio intervalo de confianza que da idea de la gran dispersión, por lo que este resultado debe tomarse con cautela. Esto podría coincidir con los resultados de otros estudios, como el de Piecuch, et al. ${ }^{5}$, en los que la patología renal se asoció con un aumento de las complicaciones de la cirugía colorrectal. La existencia de insuficiencia renal produce una disminución de la concentración de albúmina en la sangre (elemento esencial en el proceso de cicatrización), y este déficit actúa desfavorablemente en la evolución de la anastomosis intestinal. Además, los pacientes con insuficiencia renal suelen asociar comorbilidad que hace que el nivel ASA aumente, y son varios los estudios que cuentan este factor como de riesgo para el desarrollo de dehiscencia ${ }^{2-5}$.

La transfusión preoperatoria ha resultado ser un factor protector débil ante el desarrollo de una dehiscencia anastomótica en nuestro análisis. Este ha sido un hallazgo insospechado y sin respaldo en la literatura consultada, ya que al comparar este resultado con el obtenido en otros trabajos se observa que, en su mayoría, no consideran esta variable como tal, sino que se limitan a medir la existencia de anemia preoperatoria $^{6-12}$. Esta última variable, determinada por un valor de hemoglobina $<8 \mathrm{~g} / \mathrm{dl}$ en nuestra serie ${ }^{9,10}$, está asociada como factor de riesgo de complicaciones posoperatorias tras cirugía no cardiaca en diversos estudios. Sin embargo, en el nuestro, contradictoriamente, no se asocia al desarrollo de dehiscencia de la anastomosis. Lo que podemos determinar con este resultado es que la corrección de la anemia preoperatoria disminuirá el riesgo de isquemia transitoria durante la intervención, y por ello habrá una buena evolución en la cicatrización de la herida.

En un estudio realizado en la Universidad de California-Irvine ${ }^{10}$ se concluyó que la desnutrición preoperatoria, medida como cifras de albúmina sérica $<3.5 \mathrm{~g} / \mathrm{dl}$, era un factor de riesgo para la dehiscencia de la anastomosis. Varios trabajos, como el de Telem, et al. ${ }^{3}$, lo avalan. En nuestro estudio, esta variable no ha resultado ser estadísticamente significativa; sin embargo, por ser un factor fácilmente recuperable mediante suplementos proteicos o seguimientos conjuntos por parte de la unidad de nutrición, consideramos que debe tenerse en cuenta al cuantificar los factores de riesgo de dehiscencia de sutura.

En cuanto a los factores dependientes de la intervención, el tiempo operatorio alargado más allá de $180 \mathrm{mi}-$ nutos ha demostrado ser un factor de riesgo para el desarrollo de dehiscencia de anastomosis tras la cirugía de cáncer de colon en nuestra serie. Este resultado es igual al obtenido en diversos estudios de similares características. Así, los estudios de Midura, et al. ${ }^{4}$, Suding, et al. ${ }^{9}$ y Mäkelä, et al. ${ }^{13}$ asociaron la excesiva duración de la cirugía con el desarrollo de dehiscencia de anastomosis por la exposición de las vísceras al ambiente, la deshidratación de los tejidos y la contaminación por patógenos ambientales. Estudios como el de Lai, et al. ${ }^{14}$ vinculan el tiempo anestésico prolongado a una mayor probabilidad en la aparición de filtraciones anastomóticas, debido a que un aumento en la duración de la cirugía puede reflejar complicaciones intraoperatorias, presencia de dificultades anatómicas, condiciones técnicas adversas que obstaculizan las maniobras o incluso inexperiencia por parte del cirujano, haciendo que el procedimiento quirúrgico final no se desarrolle en las condiciones idóneas. Midura, et al. ${ }^{4}$, en un estudio con 13,684 pacientes realizado en los EE.UU., relacionaron la dehiscencia de la anastomosis con otros factores además del tiempo de intervención, como el sexo masculino, el uso de esteroides, el tabaquismo, el abordaje abierto y la quimioterapia previa.

Otro de los factores de riesgo reconocidos para la dehiscencia de la anastomosis es la altura de esta, en especial cuando se analiza la anastomosis colorrectal. El estudio de Vignali, et al..$^{15}$ con 1014 pacientes demostró que las anastomosis de carácter bajo están asociadas a un mayor riesgo de dehiscencia. Los estudios de Cong, et al. ${ }^{16}$ y Pakkastie, et al. ${ }^{17}$ determinan que la anastomosis a menos de $5 \mathrm{~cm}$ del ano aumenta considerablemente el riesgo de dehiscencia. La mayoría de los estudios que intentan investigar los factores de riesgo en este campo coinciden también en que la localización derecha o izquierda de la anastomosis es un factor asociado al riesgo de complicaciones en el posoperatorio. Tradicionalmente se ha considerado que la hemicolectomía derecha, por su aparente sencillez técnica, su mejor exposición del campo quirúrgico y la menor tensión en la anastomosis ileocólica, debería asociarse a un menor número de complicaciones ${ }^{18}$. En nuestro estudio hemos evitado incluir pacientes intervenidos de cáncer de recto o con cualquier estoma protector, y finalmente no se ha encontrado asociación entre la localización del tumor (colon derecho o izquierdo) y el desarrollo de dehiscencia de la anastomosis. Otros estudios respaldan este resultado ${ }^{18}$.

En los últimos años se han realizado varios estudios clínicos que avalan la eficacia y la seguridad de la vía laparoscópica en la cirugía de colon ${ }^{4,19.20}$. Este factor no ha resultado ser estadísticamente significativo en nuestro estudio, como tampoco lo es en los estudios consultados. La introducción de nuevas vías de 
abordaje, como la laparoscopia, aporta evidentes ventajas tanto clínicas como económicas y gerenciales.

En nuestro modelo, tras incluir inicialmente en la regresión logística multivariante las nueve variables seleccionadas por su importancia en la literatura, solo consiguieron mantenerse en la ecuación tres de ellas: la duración de la cirugía, la creatinina sérica y la transfusión preoperatoria. Las variables restantes fueron excluidas de la ecuación durante el proceso de selección automática por pasos hacia atrás (backward). La proporción de la variabilidad de la variable dehiscencia de la sutura que es explicada por este modelo no es demasiado buena (Tabla 2): entre un 14.4\% (según la R cuadrado de Cox y Snell) y un $19.7 \%$ (según la R cuadrado de Nagelkerke); esto es, sigue existiendo un porcentaje importante de «influencia» sobre el hecho de tener una dehiscencia de sutura que no depende de las variables analizadas e incluidas en el modelo.

\section{Conclusiones}

Un tiempo de intervención mayor de 180 minutos es un factor de riesgo para el desarrollo de dehiscencia de la anastomosis en nuestro medio. La ecuación predictiva elaborada de basándonos en nuestros resultados no explica un porcentaje importante de «influencia» sobre la aparición de dehiscencia de la anastomosis, que no depende de las variables analizadas e incluidas en el modelo. Por tanto, no resulta de utilidad en la práctica clínica y son necesarios estudios más amplios para dar respuesta a los resultados contradictorios hallados en este estudio, tratando de validar nuestra ecuación en otra población.

\section{Financiación}

El estudio ha sido realizado sin financiación.

\section{Conflicto de intereses}

Los autores declaran no tener ningún conflicto de intereses.

\section{Contribuciones de autoría}

Concepción y diseño del trabajo, recogida de datos, análisis e interpretación de los datos: MVR, FMGV y MJGR.

Escritura del artículo o revisión crítica con importantes contribuciones intelectuales: MVR, FMGV y MJGR.
Aprobación de la versión final para su publicación: MVR, FMGV y MJGR.

\section{Responsabilidades éticas}

Protección de personas y animales. Los autores declaran que para esta investigación no se han realizado experimentos en seres humanos ni en animales.

Confidencialidad de los datos. Los autores declaran que han seguido los protocolos de su centro de trabajo sobre la publicación de datos de pacientes.

Derecho a la privacidad y consentimiento informado. Los autores declaran que en este artículo no aparecen datos que identifiquen al paciente.

\section{Bibliografía}

1. Leichtle SW, Mouawad NJ, Welch KB, Lampman RM, Cleary RK. Risk factors for anastomotic leakage after colectomy. Dis Colon Rectum. 2012;55:569-75.

2. Alves A, Panis $Y$, Mathieu P, Mantion G, Kwiatkowski F, Slim K. Association Française de Chirurgie. Postoperative mortality and morbidity in French patients undergoing colorectal surgery: results of a prospective multicenter study. Arch Surg. 2005;140:278-83.

3. Telem D, Chin E, Nguyen S, Divino C. Risk factors for anastomotic leak following colorrectal surgery. Arch Surg. 2010;145:371-6.

4. Midura EF, Hanseman D, Davis BR, Atkinson SJ, Abbott DE, Shah SA, et al. Risk factors and consequences of anastomotic leak after colectomy: a national analysis. Dis Colon Rectum. 2015;58:333-8.

5. Piecuch J, Wiewiora M, Jopek J, Szrot M, Mazur I, Zurawinski W, et al. Mortality and anastomotic leakage after anterior resection for rectal cancer. Hepatogastroenterology. 2012;59:721-3.

6. Sobin $\mathrm{H}$, Wittekind $\mathrm{CH}$. TNM Classification of malignant tumours. International Union Against Cancer. New York: John Wiley and Sons; 2002.

7. Neutzling CB, Lustosa SA, Proenca IM, da Silva EM, Matos D. Stapled versus handsewn methods for colorectal anastomosis surgery. Cochrane Database Syst Rev. 2012;(2):CD003144.

8. Dunne JR, Malone D, Tracy JK, Gannon C, Napolitano LM. Perioperative anemia: an independent risk factor for infection, mortality, and resource utilization in surgery. J Surg Res. 2002;102:237-44.

9. Suding $\mathrm{P}$, Jensen $\mathrm{E}$, Abramson MA, Itani K, Wilson SE. Definitive risk factors for anastomotic leaks in elective open colorectal resection. Arch Surg. 2008;143:907-11.

10. Wu WC, Schifftner TL, Henderson WG. Preoperative hematocrit levels and postoperative outcomes in older patients undergoing noncardiac surgery. JAMA. 2007;297:2481-8.

11. Parker MC. Epidemiology of adhesions: the burden. Hosp Med. 2004; 65:330-6.

12. Kirchhoff $P$, Clavien PA, Hahnloser D. Complications in colorectal surgery: risk factors and preventive strategies. Patient Saf Surg. 2010;4:5.

13. Mäkelä JT, Kiviniemi H, Laitinen S. Risk factors for anastomotic leakage after left-sided colorectal resection with rectal anastomosis. Dis Colon Rectum. 2003;46:653-60

14. Lai R, LuY, Li Q, Guo J, Chen G, Zeng W. Risk factors for anastomotic leakage following anterior resection for colorectal cancer: the effect of epidural analgesia on occurrence. Int J Colorectal Dis. 2013;28:485-92.

15. Vignali A, Fazio VW, Lavery IC, Milson JW, Church JM, Hull TL, et al. Factors associated with the occurrence of leaks in stapled rectal anastomoses: a review of 1014 patients. J Am Coll Surg. 1997;185:105-13.

16. Cong ZJ, Fu CG, Yu ED, Liu LJ, Zhang W, Meng RG, et al. Factors associated with anastomotic leakage after anterior resection in rectal cancer. Zhonghua Wai Ke Za Zhi. 2009;47:594-8.

17. Pakkastie $T$, Luukkonen $P$, Jarvinen J. Anastomotic leakage after anterior resection of the rectum. Eur J Surg. 1994;160:293-7.

18. Groene SA, Chandrasekera CV, Prasad T, Lincourt AE, Todd Heniford B, Augenstein VA. Right versus left-sided colectomies: a comparison of outcomes. Am Surg. 2016;82:580-7.

19. Breukink S, Pierie J, Wiggers T. Laparoscopic versus open total mesorectal excision for rectal cancer. Cochrane Database Syst Rev. 2006;(4):CD005200

20. Schwenk W, Haase O, Neudecker J, Müller JM. Short term benefits for laparoscopic colorectal resection. Cochrane Database Syst Rev. 2005; (3):CD003145. 